\title{
Guaifenesin as a Treatment for Primary Dysmenorrhea
}

\author{
Jennifer S. Marsden, MD, Charlene D. Strickland, BSN, RN, and \\ MAJ Tina L. Clements, AN, USA
}

Background: Dysmenorrhea is highly prevalent and causes much work loss and discomfort. A treatment with a new mechanism of action could benefit women of menstruating age. A study was undertaken to assess the efficacy of guaifenesin as a treatment for primary dysmenorrhea because of its effects of cervical dilation and cervical mucous thinning.

Methods: Thirty-four subjects with primary dysmenorrhea were enrolled in a double-blind, placebocontrolled study. Three treatment surveys measured 10 symptoms (lower abdominal pain, general abdominal pain, back pain, headache, nausea, diarrhea, constipation, menstrual flow, weakness, and activities of daily living) on a 100-mm visual analog scale. Nonstudy analgesic use was also measured.

Results: Twenty-five subjects returned the first treatment survey, and 17 returned all 3 surveys. Results were nonsignificant, but guaifenesin trended toward being better than placebo for dysmenorrhea pain and associated constitutional symptoms and caused no worsening of symptoms. Lower abdominal mean pain scores from the first survey decreased $38 \mathrm{~mm}$ for guaifenesin versus $7 \mathrm{~mm}$ for placebo. By the third survey, only 2 of 8 guaifenesin participants took nonstudy analgesics compared with all 9 placebo subjects.

Conclusions: Guaifenesin may be useful in the treatment of primary dysmenorrhea. A larger study is needed to validate these initial findings. (J Am Board Fam Pract 2004;17:240-6.)

Dysmenorrhea is a disorder characterized by lower abdominal pain that occurs during menstruation, but the pain may start 2 or more days before menstruation. It is sometimes associated with headache, nausea, vomiting, diffuse abdominal pain, backache, general malaise, weakness, and other gastrointestinal symptoms. Dysmenorrhea is often undertreated because physicians are not fully aware of its high prevalence and morbidity. ${ }^{1}$ Primary dysmenorrhea is generally believed to affect $50 \%$ of menstruating women, ${ }^{2}$ and some degree of dysmenorrhea may be present in as many as $90 \%{ }^{1,3}$ Many women do not consider dysmenorrhea noteworthy

Submitted, revised, 15 December 2003*.

From the Department of Family and Community Medicine, Darnall Army Community Hospital, Fort Hood, Texas (TLC), and the Fort Hood Chapter, American Red Cross (JSM, CDS). Address correspondence to Jennifer S. Marsden, MD, 16 Trenchard Road, Andover, Hampshire SP11 8HB, UK (E-mail: skydoc21@yahoo.com).

This study was supported by the Department of Family and Community Medicine, Darnall Army Community Hospital, Fort Hood, Texas, and by the Fort Hood chapter of the American Red Cross. No outside funding from any source was received during completion of the project or preparation of the manuscript. The opinions or assertions contained herein are the private views of the authors and are not to be construed as reflecting the opinion of the Department of the Army or the Department of Defense. enough to answer positively about it or to bring it up with their physician even if it restricts their daily activities, yet a great deal of absenteeism and decreased productivity is caused by dysmenorrhea, and some patients are not using adequate doses of treatment. ${ }^{1}$

Dysmenorrheic women may have contractions reaching intrauterine pressures of $200 \mathrm{~mm} \mathrm{Hg}$ (versus $100 \mathrm{~mm} \mathrm{Hg}$ for a nondysmenorrheic woman) at very frequent disorganized intervals. Uterine arterial pulsations disappear, suggesting ischemia in the dysmenorrheic uterus. ${ }^{2}$ Transdermal glyceryl trinitrate decreases uterine pain., ${ }^{4,5}$ Decreasing intrauterine pressure can be achieved through decreasing the frequency and strength of uterine contractions and/or decreasing cervical os resistance.

For many women, current therapies offer inadequate treatment. Estimates of the effectiveness of current treatments (including oral contraceptives and nonsteroidal anti-inflammatory drugs) range from 64 to $90 \%$ of patients ${ }^{1}$ but some women have intolerable side effects (such as gastric upset and infertility). The available treatments decrease impairment but not to the nonmenstruating level of productivity for all women. Some patients resort to surgical treatment. The long-term and associated 
health risks of dysmenorrhea have not been studied. Adding a medication with a different mechanism of action to the treatment of dysmenorrhea may benefit some women.

Guaiacol glyceryl ether (guaifenesin) has been used for years to promote expectoration of secretions in patients with pulmonary disease. The drug is derived from the resin of guaiacum trees and was introduced to European medicine in the 16th century as an analgesic. ${ }^{6}$ Guaifenesin is also used as a muscle relaxant in veterinary anesthesia to aid in induction. ${ }^{7}$ It has been reported to be effective for prostatitis, seminal vesiculitis, benign prostatic hypertrophy, prostatic abscess, bladder neck hypertrophy, and urinary tract infection pain. ${ }^{8}$ A European patent application claims guaiacol and its derivatives are useful for the treatment of heartburn and constipation. ${ }^{9}$

Guaifenesin has also been shown to have effects on the uterine cervix. Guaifenesin (200 mg) 3 times a day improves fertility; perhaps by improving spinnbarkheit, ferning, and cellularity of cervical mucus. ${ }^{10-12}$ The 2 types of cells that produce this mucus are muciparous, similar to those found in other mucus epithelia, and other mucus cells that respond under the stimulus of ovarian hormones. ${ }^{13}$ Three obstetric articles mention the use of a guaifenesin compound in labor to speed the effacement of the cervix. ${ }^{14,15,16}$ In a study using 1000 to $2000 \mathrm{mg}$ of guaifenesin intravenously, the mean labor time was reduced by half in both primiparas and multiparas compared with the control group. ${ }^{14}$

Compounds of guaifenesin with paracetamol, caffeine, phenobarbital, or acetylsalicylic acid are used for pain control. The guaifenesin is thought to speed absorption of the other ingredients. ${ }^{17}$ Dolmina, a drug consisting of $200 \mathrm{mg}$ of guaifenesin and $325 \mathrm{mg}$ of acetylsalicylic acid per tablet, was compared with an indomethacin product in a study of women with primary dysmenorrhea. Although the difference in pain scores was insignificant, there was a strong patient preference for the guaifenesin compound. ${ }^{18}$

A patient with severe dysmenorrhea was tried on prescription dose guaifenesin (2400 mg/day) during menses, aiming for a decrease in cervical os resistance via the thinning effect of the guaifenesin on cervical mucus. She experienced resolution of her symptoms.

We hypothesized that guaifenesin alone, through its possible effects of analgesia, muscle relaxation, cervical dilation, and cervical mucous thinning, could be effective in the treatment of primary dysmenorrhea.

\section{Materials and Methods}

Subjects were recruited from December 1999 to December 2000 through newspaper and poster advertisements and physician referrals to the Fort Hood, Texas, Family Practice Clinics. Data collection continued through February 2001. Criteria for inclusion in the study were as follows: female, age between 18 and 45, not pregnant or trying to conceive, no history of adverse reaction to guaifenesin, not using oral contraceptives, nonsmoker, not breast-feeding, meeting diagnostic criteria for primary dysmenorrhea (no diagnosis of secondary dysmenorrhea), having moderate to severe symptoms when unmedicated, and eligible for care through the military health system. Informed consent was obtained from all participants.

The research protocol, consent forms, and surveys were approved by the Brooke Army Medical Center Institutional Review Board (study C-00003). Procedures followed were in accordance with the ethical standards for human experimentation established by the Declaration of Helsinki of 1975 , revised in 1983.

Two of the investigators enrolled all the subjects. The medication used was long-acting $600-\mathrm{mg}$ guaifenesin. Both placebo and drug were in identical capsules. The Chief of the Outpatient Pharmacy used computer-generated random numbers to make coded assignments to the placebo or drug group, stratified into 20 subject blocks. Subjects were given numbered bottles based on the order of their appearance at the pharmacy. All the investigators, participants, and support staff, including the dispensing pharmacists were blinded to the group assignment until after completion of enrollment.

Subjective symptom scores and subject-reported dosing amounts and frequencies were solicited. The investigators developed a $100-\mathrm{mm}$ visual ana$\log$ scale menstrual questionnaire with 5 time intervals: first 2 hours (from onset) of menses, 2 to 12 hours, 12 to 24 hours, second day, and third day. Anchors were labeled 'no pain' or no symptoms and 'extreme pain' (or other extreme) of the 10 symptoms: lower abdominal pain, general abdominal pain, back pain, headache, nausea, diarrhea, constipation, menstrual flow, weakness/feeling faint, and 
Table 1. Characteristics of Enrolled Subjects and of Subjects Returning the First Treatment Survey

\begin{tabular}{|c|c|c|c|c|}
\hline \multirow[b]{2}{*}{ Subject Characteristics } & \multicolumn{2}{|c|}{ Enrolled Initially* } & \multicolumn{2}{|c|}{ Returned Survey $1^{*}$} \\
\hline & Guaifenesin & Placebo & Guaifenesin & Placebo \\
\hline Total subjects & 18 & 16 & 13 & 12 \\
\hline Age, mean years (SD) & $32.3(7)$ & $32.2(7.1)$ & $32.3(7.4)$ & $33.9(6.5)$ \\
\hline Menarche, mean years (SD) & $12.2(1.9)$ & $12.6(2.2)$ & $11.8(2.0)$ & $13.2(2.1)$ \\
\hline Menstrual cycle length, mean days ${ }^{\dagger}(\mathrm{SD})$ & $28.1(4.3)$ & $26.6(3.7)$ & $27.0(4.0)$ & $27.4(2.3)$ \\
\hline Weight, mean pounds (SD) & $178.3(41.5)$ & $156.5(34.9)$ & $176.3(42.5)$ & $152.7(35.8)$ \\
\hline Mean gravidity (SD) & $1.8(1.5)$ & $1.4(1.1)$ & $1.6(1.3)$ & $1.4(1.0)$ \\
\hline Infertility problems & $5(28 \%)$ & $3(19 \%)$ & $4(31 \%)$ & $2(17 \%)$ \\
\hline Bilateral tubal ligation rate & 0.5 & 0.3 & 0.4 & 0.3 \\
\hline Age pain onset, mean years (SD) & $18.2(7.7)$ & $18.6(6.8)$ & $19.2(8.8)$ & $19.2(6.4)$ \\
\hline Work loss taking usual medication, mean days ${ }^{\ddagger}$ & 0.5 & 0.25 & 0.31 & 0.25 \\
\hline Work loss taking no medication, mean days ${ }^{\ddagger}$ & 1.11 & 1.03 & 0.92 & 1.13 \\
\hline
\end{tabular}

* No significant differences between drug and placebo groups using the $t$ test (two-sample assuming equal variances).

${ }^{\dagger}$ Used middle number of range they chose.

‡ Prestudy

daily activities. The survey also requested information about side effects, overall satisfaction with the study medication (a single $100-\mathrm{mm}$ visual analog scale for the entire cycle/questionnaire with anchors 'not at all pleased' and 'excellent'), any nonstudy drug use, daily amount of study drug taken, and any health changes (and instructions to telephone for any new health problems or reactions).

At enrollment, each subject completed an initial health history and 2 menstrual questionnairesone about their most recent (with their usual pain medications) menses and one about their most recent unmedicated menses with symptoms, if they could recall any. Subjects were then given 3 men- strual questionnaires to be completed during their next 3 menstrual cycles, the prescription for the study medication, and written instructions for medication dosing and returning the questionnaires.

Nonstudy analgesic use was categorized as "none," "minimal," or "high" by classifying each subject's reported amount of nonstudy medication intake on days 1 and 2 of their menses. The "none" category was defined as no reported nonstudy drug use on that day. The "minimal" category was defined as a subject using no more than $800 \mathrm{mg} /$ day of ibuprofen, $300 \mathrm{mg} /$ day of naproxen, $1250 \mathrm{mg} /$ day of acetaminophen, or an equivalent combination of analgesics. The "high" category was defined as a

Table 2. Nonstudy Analgesic Use Reported by Sample

\begin{tabular}{|c|c|c|c|c|c|c|}
\hline & \multicolumn{2}{|c|}{ None* } & \multicolumn{2}{|c|}{ Minimal $^{\dagger}$} & \multicolumn{2}{|c|}{$\operatorname{High}^{\ddagger}$} \\
\hline & $\begin{array}{c}\text { Guaifenesin } \\
\mathrm{N}(\%)\end{array}$ & $\begin{array}{l}\text { Placebo } \\
\text { N (\%) }\end{array}$ & $\begin{array}{c}\text { Guaifenesin } \\
\text { N (\%) }\end{array}$ & $\begin{array}{l}\text { Placebo } \\
\text { N (\%) }\end{array}$ & $\begin{array}{c}\text { Guaifenesin } \\
\text { N }(\%)\end{array}$ & $\begin{array}{c}\text { Placebo } \\
\text { N (\%) }\end{array}$ \\
\hline \multicolumn{7}{|l|}{ Trial 1} \\
\hline Day 1 & $6(46 \%)$ & $4(33 \%)$ & $4(31 \%)$ & $4(33 \%)$ & $3(23 \%)$ & $4(33 \%)$ \\
\hline Day 2 & $10(77 \%)$ & $5(42 \%)$ & $2(15 \%)$ & $3(25 \%)$ & $1(8 \%)$ & $4(33 \%)$ \\
\hline \multicolumn{7}{|l|}{ Trial 2} \\
\hline Day 1 & $6(55 \%)$ & $1(10 \%)$ & $2(18 \%)$ & $3(10 \%)$ & $3(27 \%)$ & $6(60 \%)$ \\
\hline Day 2 & $8(73 \%)$ & $3(30 \%)$ & $2(18 \%)$ & $3(30 \%)$ & $1(9 \%)$ & $4(40 \%)$ \\
\hline \multicolumn{7}{|l|}{ Trial 3} \\
\hline Day 1 & $6(75 \%)$ & $1(11 \%)$ & $2(25 \%)$ & $5(56 \%)$ & $0(0 \%)$ & $3(33 \%)$ \\
\hline Day 2 & $6(75 \%)$ & $0(0 \%)$ & $2(25 \%)$ & $5(56 \%)$ & $0(0 \%)$ & $4(44 \%)$ \\
\hline
\end{tabular}

* No reported nonstudy analgesic medication used.

${ }^{\dagger}$ Nonstudy analgesic use reported to be no more than $800 \mathrm{mg} /$ day of ibuprofen, $300 \mathrm{mg} / \mathrm{day}$ of naproxen, $1250 \mathrm{mg} / \mathrm{day}$ of acetaminophen, or an equivalent combination of analgesics.

${ }^{\ddagger}$ Nonstudy analgesic use reported to be any combination of more than $800 \mathrm{mg} /$ day of ibuprofen, $300 \mathrm{mg} /$ day of naproxen, or 1250 $\mathrm{mg} /$ day of acetaminophen. 
subject using any combination of more than 800 $\mathrm{mg}$ /day of ibuprofen, $300 \mathrm{mg} /$ day of naproxen, or $1250 \mathrm{mg} /$ day of acetaminophen.

The raw scores for each time interval and symptom were evaluated along with pain differential scores subtracted from their baseline surveys (with their usual pain medication or unmedicated). All the surveys returned were included in the analysis regardless of whether the subject reported compliance with the study medication. An additional analysis of a subgroup of participants who reported compliance with the prescribed dose of study medication and who did not report any nonstudy analgesic use (for scores from the first 2 hours in the first survey) was also performed.

Subjects were asked to take 4 capsules $(2400 \mathrm{mg})$ per day over the first 2 days ( 8 capsules total) and were asked to report the number of study medication capsules taken each day for each menstrual cycle. Compliance with the study medication was stratified by categorizing surveys as compliant if subjects took 7 to 8 capsules, partly compliant if they took 3 to 6 , and noncompliant if they took 0 to 2 capsules. Compliance was also assessed as the subject having taken the prescribed 2 capsules by the second hour of menses to correlate scores from the first 2 hours with actual medication use.

Response to treatment rates (defined as $50 \%$ or better drop from the most recent menses scores using usual medications baseline as denominator) were calculated for the lower abdominal pain scores.

A power analysis was performed. Detecting a medium effect $(0.5 \mathrm{SD})$ required 97 subjects per group (194 total). Detecting a large effect (0.8 SD) required 40 subjects per group (80 total). Subject characteristics were compared using the $t$ test (2-sample assuming equal variances) from the Microsoft Excel data analysis package.

\section{Results}

Thirty-four women enrolled in this study between December 1999 and December 2000. Their ages ranged from 18 to 43 . The total prestudy work loss was 36.5 days per cycle for 33 unmedicated subjects and 13 days total for 32 subjects with their usual medication. One subject could not recall an unmedicated cycle, and 2 subjects did not reply about disability on their usual medication. Only 5 women reported no disability while taking their usual med-
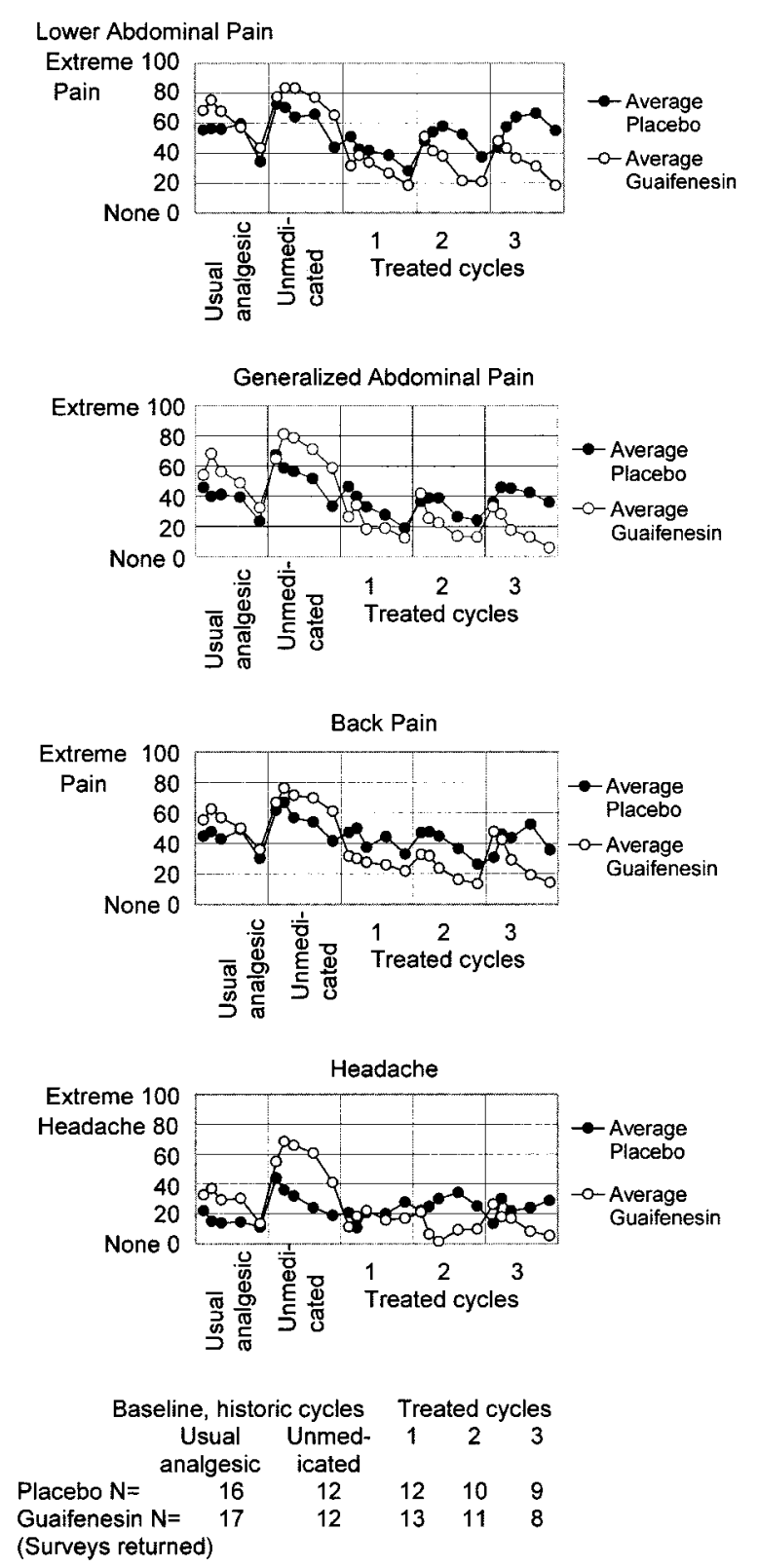

Figure 1. Mean pain scores for usual analgesic baseline, unmedicated baseline, and treatment cycles 1,2 , and 3. In each cycle, the data points are the for the first 2 hours, from 2 to 12 hours, from 12 to 24 hours, the second day, and the third day of the menses. The table at the bottom lists the number of surveys received for each cycle to highlight the study dropout rate.

ication, and 18 missed no work but reported decreased productivity. We analyzed the characteristics of the drug and placebo groups to rule out heterogeneity using the $t$ test, and there were no significant differences (Table 1). 

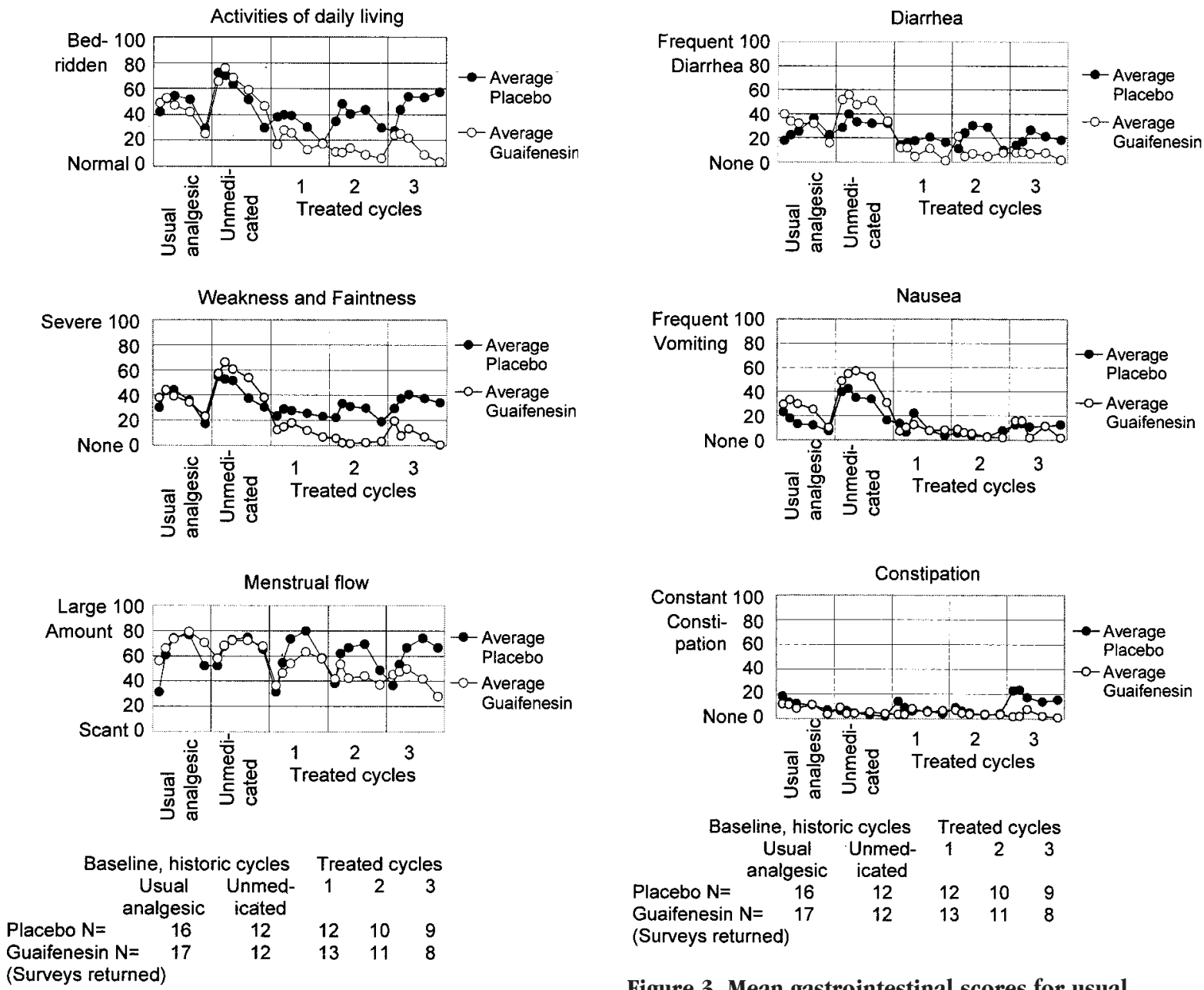

Figure 2. Mean constitutional and menstrual blood flow scores for usual analgesic baseline, unmedicated baseline, and treatment cycles 1, 2, and 3. In each cycle, the data points are for the first 2 hours, from 2 to 12 hours, from 12 to 24 hours, the second day, and the third day of the menses. The table at the bottom lists the number of surveys received for each cycle to highlight the study dropout rate.

Nine women dropped out of the study before returning any surveys during treatment; 4 dropped out after returning 1 survey; and 4 additional subjects dropped out after returning 2 surveys. The majority of subjects that dropped out had moved or changed phone numbers and could not be reached, a frequent occurrence for military personnel and their family members. The dropout rate was essentially equivalent between the placebo and guaifenesin group throughout the study. Eight drug and 9 placebo subjects returned all 3 surveys.

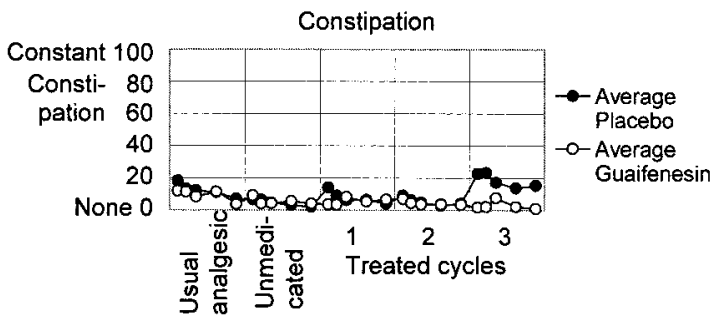

\begin{tabular}{llccccc} 
& $\begin{array}{c}\text { Baseline, historic cycles } \\
\text { Usual }\end{array}$ & \multicolumn{4}{c}{ Treated cycles } \\
annalgesic & $\begin{array}{c}\text { Unmed- } \\
\text { icated }\end{array}$ & 1 & 2 & 3 \\
Placebo N= & 16 & 12 & 12 & 10 & 9 \\
Guaifenesin N= & 17 & 12 & 13 & 11 & 8 \\
(Surveys returned) & & & & &
\end{tabular}

Figure 3. Mean gastrointestinal scores for usual analgesic baseline, unmedicated baseline, and treatment cycles 1, 2, and 3. In each cycle, the data points are for the first 2 hours, from 2 to 12 hours, from 12 to 24 hours, the second day, and the third day of the menses. The table at the bottom lists the number of surveys received for each cycle to highlight the study dropout rate.

The number of participants who completed the study was well below the sample size estimated for adequate statistical power. Thus, meaningful statistical calculations on the data could not be conducted. The dwindling enrollment after 1 year and the relocation of the principal investigator forced the termination of the study before the goal of 80 to 200 subjects was achieved. However, clinically important trends between the groups are described below.

There was a trend for greater nonstudy analgesic use in the placebo group than in the guaifenesin group, and this was more notable as the study progressed (Table 2). The variety of nonstudy 
Table 3. Overall Satisfaction with Taking Study Medication, Mean Score* on 100-mm Visual Analog Scale

\begin{tabular}{lccc}
\hline & Trial 1 & Trial 2 & Trial 3 \\
\hline Guaifenesin & 41.2 & 38.0 & 35.0 \\
Placebo & 33.3 & 25.0 & 22.3 \\
\hline
\end{tabular}

* 0 , not at all pleased; 100, excellent.

analgesic medications used included ibuprofen, naproxen, acetaminophen, propoxyphene (Darvon) and combinations such as Midol and Pamprin. Subjects who used a nonstudy analgesic drug for some purpose other than treating symptoms of dysmenorrhea (eg, ibuprofen for plantar fasciitis in the guaifenesin group, and amitriptyline and sertraline in the placebo group) were classified as minimal use unless their other analgesic use qualified them as high use (eg, fluoxetine in the placebo group). During the third survey, none of the guaifenesin subjects were classified as 'high use' and 6 of 8 guaifenesin subjects used no nonstudy analgesic. In contrast, all placebo subjects used some nonstudy analgesic during the third survey.

Figures 1, 2, and 3 show raw symptom score means for each symptom, cycle, and study time interval. The baselines (historic, prestudy) are the 'Usual analgesic' and 'Unmedicated' cycles. There is a trend toward improved symptom scores and no trend toward worsening of any symptom for the guaifenesin group compared with baseline and placebo. Table 3 shows the overall satisfaction scores. Response rates (defined as a $\geq 50 \%$ drop in pain scores from baseline) are shown in Table 4.

Subtracting from the most recent, medicated cycle as a baseline (available for all but one subject), the lower abdominal mean pain scores were
-38.1 for guaifenesin versus -6.8 for placebo at hour 2 of the first survey $(\mathrm{N}=12$ in the drug group and 12 in the placebo group). In the subset of subjects without nonstudy drug use and compliant for study medication, the guaifenesin group had a lower abdominal pain score decrease of -47.4 versus the decrease in the placebo group of $-9.1(\mathrm{~N}=10$ in the drug group and 11 in the placebo group).

\section{Discussion}

This study suggests that guaifenesin may be a better treatment than placebo for dysmenorrhea pain and associated constitutional symptoms, weakness, faintness, and limitations of activities of daily living. It does not show any trend toward worsening of any of the evaluated symptoms with guaifenesin.

The conclusions that can be drawn from this study are limited because of its small size and the confounding use of nonstudy analgesics. There was also a high dropout rate and possible recall bias, especially for the baseline symptom scores. The generalizability of the findings may be questioned because all the subjects had access to low-cost or free health care, along with a steady income (both provided by the military). In addition, they all lived in the Fort Hood area, all were soldiers or family members of soldiers, and most received their health care on the military base.

The study finding of a reduction in the placebo response over the treatment cycles is consistent with a study of placebo response in dysmenorrhea that found a favorable response to placebo of $84 \%$ in the first trial, and then $29 \%, 16 \%$, and $10 \%$ in the successive trials. ${ }^{19}$ The findings are also consistent with a study of intrauterine pressures in dysmenorrheic patients: the initial pressure reductions in the placebo group were not sustained, whereas

Table 4. Response Ratio of Subjects with $\geq 50 \%$ Decrease in Lower Abdominal Pain Score from Medicated Baseline Menses at Each Study Time Interval

\begin{tabular}{|c|c|c|c|c|c|c|}
\hline \multirow[b]{2}{*}{$\begin{array}{l}\text { Time from Onset } \\
\text { of Menses }\end{array}$} & \multicolumn{2}{|c|}{ Trial 1} & \multicolumn{2}{|c|}{ Trial 2} & \multicolumn{2}{|c|}{ Trial 3} \\
\hline & $\begin{array}{l}\text { Guaifenesin } \\
\text { Ratio (\%) }\end{array}$ & $\begin{array}{c}\text { Placebo } \\
\text { Ratio (\%) }\end{array}$ & $\begin{array}{l}\text { Guaifenesin } \\
\text { Ratio (\%) }\end{array}$ & $\begin{array}{c}\text { Placebo } \\
\text { Ratio (\%) }\end{array}$ & $\begin{array}{l}\text { Guaifenesin } \\
\text { Ratio (\%) }\end{array}$ & $\begin{array}{l}\text { Placebo } \\
\text { Ratio (\%) }\end{array}$ \\
\hline Hours 0 to 2 & $6 / 12(50 \%)$ & $2 / 12(17 \%)$ & $3 / 10(30 \%)$ & $4 / 10(40 \%)$ & 1/7 (14\%) & $3 / 9(33 \%)$ \\
\hline Hours 2 to 12 & $6 / 12(50 \%)$ & 2/11 (18\%) & 4/10 (40\%) & $3 / 9(33 \%)$ & $2 / 7(28 \%)$ & $1 / 8(13 \%)$ \\
\hline Hours 12 to 24 & $6 / 11(55 \%)$ & 4/10 (40\%) & $5 / 10(50 \%)$ & 2/9 (22\%) & 4/7 (57\%) & $1 / 8(13 \%)$ \\
\hline Second Day & $5 / 12(42 \%)$ & 4/10 (40\%) & $5 / 10(50 \%)$ & $2 / 8(25 \%)$ & $5 / 7(71 \%)$ & $1 / 8(13 \%)$ \\
\hline Third Day & $5 / 12(42 \%)$ & 4/10 (40\%) & $3 / 10(30 \%)$ & $4 / 8(50 \%)$ & $3 / 7(43 \%)$ & $2 / 8(25 \%)$ \\
\hline
\end{tabular}


pressures remained low in patients with active treatment. ${ }^{20}$ The data from the second and third surveys might be preferable to use because of the expected decrease in placebo effect, but the number responding to these surveys was quite low in this study.

Recall bias may have influenced the baseline scores. An article on recalled menstrual pain found that, in women with dysmenorrhea, pain score measurements collected 2 weeks after menses are biased to be significantly lower than scores collected at the time of the pain. ${ }^{21}$ Prospective rather than recalled baseline surveys before experimental treatment would possibly be more accurate. Thus, it is possible that the benefit of guaifenesin was underestimated. However, the compliance with taking absolutely no medication for pain during one baseline cycle would probably be low given the quantity of outside analgesic use in this study when participants were asked to use only the study drug.

This pilot study suggests that guaifenesin is a potential low-cost, low-risk treatment modality for dysmenorrhea and could be beneficial for patients unwilling or unable to tolerate current therapies, such as oral contraceptives, nonsteroidal antiinflammatory drugs, other analgesics, or surgery. Studies to further delineate guaifenesin's benefit and, if present, its mechanism of action in the treatment of dysmenorrhea may assist a large population of women not fully treated at present and could have implications for the treatment of causes for secondary dysmenorrhea, such as endometriosis.

\section{References}

1. Coco AS. Primary dysmenorrhea. Am Fam Physician 1999;60:489-96.

2. Dawood MY, McGuire JL, Demers LM, eds. Premenstrual syndrome and dysmenorrhea. 1st ed. Baltimore: Urban \& Schwarzenberg; 1985.

3. Jamieson DJ, Steege JF. The prevalence of dysmenorrhea, dyspareunia, pelvic pain, and irritable bowel syndrome in primary care practices. Obstet Gynecol 1996;87:55-58.

4. The Transdermal Nitroglycerine/Dysmenorrhoea Study Group. Transdermal nitroglycerine in the management of pain associated with primary dysmenorrhoea: a multinational pilot study. J Int Med Res 1997;25:41-4.

5. Moya RA, Moisa CF, Morales F, Wynter H, Ali A, Narancio E. Transdermal glyceryl trinitrate in the management of primary dysmenorrhea. Int J Gynaecol Obstet 2000;69:113-8.
6. Boyd EM, Sheppard EP, Boyd CE. The pharmacological basis of the expectorant action of glyceryl guaiacolate. Appl Ther 1967;9:55-9.

7. Plumb DC. Veterinary drug handbook. White Bear Lake (MN): PharmaVet Publishing; 1991. p. 116.

8. Bush IM, inventor. Method for the treatment of urologic disorders. United States patent US 4822610. 1989 Apr 18.

9. Rhodes J, Evans BK, Heatley RV. [For use of guaifenesin compounds for heartburn and constipation.] European Patent Office Bulletin 1982;82/27: pub.no. 0055313.

10. Kippley J. The art of natural family planning. 4th ed. Cincinnati: The Couple to Couple League; 1996.

11. Check JH, Adelson HG, Chung-Hsiu W. Improvement of cervical factor with guaifenesin. Fertil Steril 1982;37:707-8.

12. Check JH. Successful pregnancy despite advanced age and elevated serum follicle stimulating hormone levels-a case report. Clin Exp Obstet Gynecol 2000;27:171-2.

13. Faccioli G. Hormone dependence of human cervical mucus and variability of mucus canalization and crystallization during the menstrual cycle. Acta Eur Fertil 1984;15:43-9.

14. Auclair JM, Anton JP, Bellocq J, Zylberberg B. [Study of the action of the association of glyceric ether of guaiacol and camylofine on the dilatation of the cervix during the labor]. Rev Fr Gynecol Obstet 1970;65:523-8.

15. Anglade JP, Monteil L, Cherasse A. [Trial of an injectable antispasmodic agent, Dilavacol, during delivery (apropos of 50 cases)]. Therapie 1971;26:431-8.

16. Ratkóci I, Doszpod J, Bognár J. Effect of guaiphenesin on labour. Acta Chir Acad Sci Hung 1967;8: 191-4.

17. Perlík F, Janku I, Kordač V. The effect of guaiphenesin on absorption and bioavailability of paracetamol from composite analgesic preparations. Int J Clin Pharmacol Ther Toxicol 1988;26:413-6.

18. Kraus I, Horský A, Presl J, et al. [Combined treatment of idiopathic dysmenorrhoea by acetylsalicylic acid and guaiphenezine.] Csek Gynekol 1981;46: 601-5.

19. Fedele L, Marchini M, Acaia B, Garagiola U, Tiengo M. Dynamics and significance of placebo response in primary dysmenorrhea. Pain 1989;36: 43-7.

20. Smith RP. Objective changes in intrauterine pressure during placebo treatment of dysmenorrhea. Pain 1987;29:59-66.

21. Brodie EE, Niven CA. Remembering an everyday pain: the role of knowledge and experience in the recall of the quality of dysmenorrhea. Pain 2000;84: 89-94. 